Acta Cryst. (1991). B47, 821-823

\title{
Editorial
}

\section{Electronic submission and publication of structural results in Acta Crystallographica}

\section{Introduction}

Crystallography has become a data-intensive science. Over the past two decades, our discipline has evolved from the study of small, simple structures to structural studies of macromolecules containing thousands of atoms. Crystal structure analysis is now an essential tool in most chemistry departments, and thousands of new crystal structures of organic and inorganic compounds are determined annually. Acta Crystallographica currently publishes the results of more than 1000 new crystal structure determinations each year. The majority of these structures are published in Section C (Crystal Structure Communications). A smaller number of new structures appear in Section B (Structural Science), where one or more structures may be discussed in depth, often in relation to existing results in the literature. In essence, the structural papers submitted to Acta Crystallographica form a continuum, from the briefest of reports through papers that discuss the structural results and implications in great detail.

Although the structural results and modes of presentation differ considerably for the various types of crystal structure papers, they all share a common need for effective and reliable procedures to handle the essential crystallographic data. The reliability demanded of crystal structures requires that crystallographic data be checked as carefully as humanly possible - which applies as much to the publication, transmission and storage as to the initial determination. Realizing the importance of data integrity, the International Union of Crystallography voted at the Perth Congress in 1987 to establish a Working Party for Crystallographic Information, with a mandate to assess how crystallographic data should be managed in the coming decade. The activities of this Working Party have been closely coordinated with new initiatives by the Commission on Journals and the Commission on Crystallographic Data, to develop improved procedures for publishing, processing and storing the results of crystal structure studies. This coordinated effort has led to the development of the Crystallographic Information File [CIF: Hall, Allen \& Brown (1991). Acta Cryst. A47, 655-685], which will become the IUCr's standard for its crystallographic publications. The CIF not only provides a simple standard format for the archiving and electronic transmission of crystallographic data, but also makes it feasible to move towards the direct submission of machine-readable manuscripts to Acta Crystallographica. Following publication, these machine-readable files may then be communicated directly to the relevant crystallographic databases.

Until now, all manuscripts were submitted as hard copy which was then used as the basis for typesetting. Although the essential information was usually stored in authors' computers, the route between manuscript preparation and the final publication in Acta Crystallographica often required repeated manual keyboarding of numerical data. Procedures for checking the data were awkward and required repeated proof-reading by authors, technical editors and typesetters. Rather than concentrating completely on the scientific content of structural papers, Co-editors of Acta Crystallographica were spending considerable effort simply trying to verify the numerical validity of crystallographic data. Understandably, errors have managed to escape these review procedures. In addition, the manual procedures involved were expensive and delayed publication of structural results. Thus, the effort to computerize our procedures for handling and publishing crystal structure results has been a major priority of the Commission on Journals for the past several years.

The first critical step toward electronic handling of crystal structure papers was the development of a standard file structure, which is now provided by the Crystallographic Information File. Full details of the CIF, together with examples and a complete dictionary of the CIF data field names are described by Hall et al. (1991). The originators of the major crystallographic software packages have been kept fully aware of CIF developments. We hope that these packages will soon provide for the automated output of crystallographic data in the new standard format. A particular feature of CIF design is the availability of data fields to record the text of the manuscript together with details of submission and of the subsequent handling by Co-editors and technical staff. This information can be added readily to an existing CIF using any simple text editor. In considering the application of the CIF to Acta Crystallographica publications, the Commission on Journals has conducted a thorough review of all procedures relating to the publication of new structural data in Sections $\mathrm{B}$ and $\mathrm{C}$. These include procedures for submission, categorization, handling, information content and printed layout in the journal.

\section{Procedures for Acta Crystallographica, Section C}

Beginning in early 1992, Acta Crystallographica, Section $C$, will adopt new procedures for the submission and publication of crystal structure papers. A revised Notes for Authors, describing these new procedures and listing the essential data items required for structural papers, appears in the November issue of Section C [Acta Cryst. (1991), C47, 2266-2275].

The fundamental goal of the new procedures is to facilitate the direct transfer of numerical data, and the associated manuscript text, from an author's computer to the typesetting programs that are used to prepare the printed 
paper. An intermediate step should involve a detailed computerized checking of the crystallographic data and structural results. We are pleased to report that automated systems to accomplish these aims have now been established by the technical staff in Chester. An input manuscript in CIF format may now be checked for numerical consistency and processed to a first printed proof before it is transmitted to a Co-editor for scientific appraisal. A major change in submission procedures, then, is that from 1 January 1992 all papers intended for Section C should now be submitted directly to the Technical Editor in Chester and not to individual Co-editors.

The Commission is well aware that the ability to submit machine-readable files, particularly via electronic mail, is not yet open to all authors. Procedures have, therefore, been devised for the continuing submission of structural papers as hard-copy manuscripts. Crystallographic Information Files will be prepared in Chester from the hard copy, so that the benefits of electronic checking and publishing can be enjoyed by the entire crystallographic community. In order to facilitate efficient handling of hardcopy manuscripts, the essential experimental data must now be encoded on the simple form which is included with the new Notes for Authors. Additionally, hard-copy submissions should be arranged according to the new Section $\mathrm{C}$ layout described below.

The revised Notes also describe the uniform printed layout that will become the standard for Section C. The somewhat arbitrary distinction between 'full articles' and 'short-format papers' is removed and the new format comprises the following sections:

Text material:

Title, Authors, Affiliations

Abstract

Comment

Acknowledgements

References

Tabulated data:

Experimental details

Atomic coordinates

Selected geometry

Illustrations:

Chemical structural
diagram
Crystallographic
diagram(s)

The information content of each section is fully defined in the revised Notes and an example of the format follows these Notes in the present issue. The most important changes from current practices are:

\section{Abstract}

This will now summarize the major structural features. It will not contain the 'crystal data' which will now be tabulated together with the other experimental details.

\section{Comment}

This section subsumes the text previously presented under 'Introduction' and 'Discussion' (for full articles) or 'Related literature' (for short-format papers). There is no formal length restriction for this Comment section. However, it should at least indicate why the analysis was performed, give the origin of the samples (if relevant), and give leading references to related work. At the upper end of the spectrum, longer Comment sections will be assessed by Co-editors and referees in the usual way and publication of particularly detailed material may be transferred to Section B (see below). It is expected that Comment material in Section C will not normally exceed a single column in the printed journal.

\section{Geometry}

The most important geometrical data should be selected for printed publication (the CIF contains the facilities for making this selection). The selection may be amended by Co-editors or referees.

\section{Chemical structural diagram}

The Commission on Journals has decided that a twodimensional chemical structural diagram should be provided for all except the simplest of structures. This diagram should show the formal chemical bond types, and is invaluable in correlating the crystallographic results with the relevant chemical features.

The changes described above will bring direct and immediate benefits to authors and readers of Section C. Submitted manuscripts will now be handled more efficiently, errors that result from repetitive keyboarding of data will be eliminated, and the printed papers can now be published more rapidly. More importantly, the overall quality of papers will be maintained and improved, not only through the extensive and consistent checking procedures, but also by allowing Co-editors to concentrate on the scientific merit and information content of each new submission.

Eventually, the IUCr should realize significant cost savings that will result from electronic publishing procedures. Authors will be strongly encouraged to submit manuscripts using the Crystallographic Information File. We expect that these new procedures will increase the throughput of manuscripts, and will enhance the accuracy of the printed contents of Section $\mathrm{C}$, and, in the near future, of Section B as well. This will serve as a logical first step toward the longer-range goal of computerizing and automating many other phases of manuscript submission, editing and publishing.

\section{Criteria for Acta Crystallographica, Section B}

In the past few years, the Commission on Journals has been concerned about the criteria for distinguishing between structural papers to be published in Section B and Section C. In the future, the purpose of Section $C$ will be to publish short communications that report the results of one or more crystal structure determinations in a compact and standardized format. Section B will continue to publish more detailed papers describing structural studies. In its briefest form, the criterion by which a paper is judged 
suitable for Section B is that 'it makes a significant contribution to a specific area of structural science'. In the past, the impression was given that a paper presenting a detailed discussion of one, or even a few, new crystal structures was not suitable for inclusion in Section B. The policy of the Commission on Journals is that such papers do fall within the general criterion stated above, and a number of papers of this type have been published in recent issues of Section B. The main factors which differentiate a Section B paper from a Section C communication are $(a)$ the length and scientific quality of the discussion material which may lead to a need for $(b)$ additional tabulated material and/or $(c)$ additional diagrams and figures.

\section{Conclusion}

The changes announced in this Editorial are of far-reaching significance for the publication and dissemination of crystallographic results. The changes are both necessary and inevitable in view of the rapid increase in crystallographic output and, more generally, in view of the technology now available for information transfer and journal production. The key to these changes is the adoption of a single common data format that is directly linked to the processes involved in journal production and printing. We realize that this transition to a common format cannot take place overnight. It can only occur with the goodwill of the crystallographic community, in terms of both software developments and a willingness to employ the new procedures. We are certain that this goodwill exists and that the ultimate benefit of these initiatives will be considerable. Indeed, as expertise in electronic publishing develops, we envision that non-structural papers will also be processed in the near future from machine-readable submissions.

These developments have required extensive work and commitment from a number of individuals. We are especially grateful to S. R. Hall and I. D. Brown for their role in developing the Crystallographic Information File, which is at the heart of our efforts to standardize the handling of crystallographic data. We are deeply indebted to the technical-editing staff in Chester for undertaking this time-consuming commitment on top of their other pressing daily responsibilities, and for establishing the computer systems and procedures necessary to accept, process and publish data based upon CIF input.

F. H. ALLEN Chairman. Commission on Crystallographic Data C. E. BUGG Chairman, Commission on Journals E. N. MASLEN Chairman, Working Party for Crystallographic Information 